\title{
Upper Bound of Partial Sums Determined by Matrix Theory
}

\author{
Rabha W. Ibrahim* \\ Institute of Mathematical Sciences, University Malaya, Malaysia \\ *Corresponding author: rabhaibrahim@yahoo.com
}

Received June 18, 2015; Revised November 25, 2015; Accepted December 03, 2015

\begin{abstract}
One of the major problems in the geometric function theory is the coefficients bound for functional and partial sums. The important method, for this purpose, is the Hankel matrix. Our aim is to introduce a new method to determine the coefficients bound, based on the matrix theory. We utilize various kinds of matrices, such as Hilbert, Hurwitz and Turan. We illustrate new classes of analytic function in the unit disk, depending on the coefficients of a particular type of partial sums. This method shows the effectiveness of the new classes. Our results are applied to the well known classes such as starlike and convex. One can illustrate the same method on other classes.
\end{abstract}

Keywords: analytic function, univalent function, unit disk, partial sums, coefficients bound

Cite This Article: Rabha W. Ibrahim, "Upper Bound of Partial Sums Determined by Matrix Theory.” Turkish Journal of Analysis and Number Theory, vol. 3, no. 6 (2015): 149-153. doi: 10.12691/tjant-3-6-2.

\section{Introduction}

The Hankel determinant represents a major part in the theory of singularities [1,2]. In addition, it utilizes in the investigation of power series with integral coefficients [3]. Also, it appears in the study of meromorphic functions [4], and various properties of these determinants can be found in [5]. It is well known that the Fekete-Szego functional $\left|a_{3}-a_{2}^{2}\right|=H_{2}(1)$. This functional is further generalized as $\left|a_{3}-\mu a_{2}^{2}\right|$ for some $\mu$ (real or complex). Fekete and Szego introduced sharp bounds of $\left|a_{3}-\mu a_{2}^{2}\right|$ for $\mu$ real of univalent functions. It is a very important combination of the two coefficients which describes the area problems posted earlier by Gronwall. Furthermore, researchers considered the functional $\left|a_{2} a_{4}-a_{3}^{2}\right|$ (see [6]). Babalola [7] determined the Hankel determinant $H_{3}(1)$ for some subclasses of analytic functions. Ibrahim [8] computed the Hankel determinant for fractional differential operator in the open unit disk.

Partial sums are studied widely in the univalent function theory. Szeg [9] proved that if the function $f(z)=z+\sum_{n=2}^{\infty} a_{n} z^{n}$ is starlike, then its partial sums $f_{k}(z)=z+\sum_{n=2}^{k} a_{n} z^{n} \quad$ are starlike for $|z|<1 / 4$. Moreover, if $f(z)$ is convex, then its partial sums $f_{k}(z)$ is convex for $|z|<1 / 8$. Later Owa [10] imposed the starlikeness and convexity for special case of $f_{k}(z)=z+a_{k} z^{k}$. In addition, Darus and Ibrahim [11] specified the assumptions, which indicated that the partial sums of functions of bounded turning are also of bounded turning. Recently, Darus and Ibrahim [12] considered the Cesáro partial sums, it has been shown that this type of partial sums preserves the properties of the analytic functions in the open unit disk.

In this work, we deal with the partial sums of the form $f_{k}(z)=z+\left(a_{k} / k\right) z^{k}, k>2$. We introduce some classes of analytic functions defined by its partial sums. The stability of these classes is studied by utilizing Hurwitz matrices convoluting the with Hilbert matrix (a special type of Hankel matrix). Moreover, we discuss some partial sums formulated under Turan determinant. The upper bound as well as the lower bound of the coefficients $a_{n}$. This new process includes some well known results. Our outcomes depend on computational results of different order of the Turan determinant. We show that some geometric properties, of the new classes are established by computing the Turan determinant such as starlikeness and convexity.

\section{Processing}

Let $\mathcal{A}$ be the class of analytic functions $f_{k}(z)=z+\sum_{n=2}^{k} a_{n} z^{n} \quad$ in $\quad U=\{z:|z|<1\} \quad$ and normalized by the conditions $f(0)=f^{\prime}(0)-1=0$. For a partial sum of the form

$$
f_{k}(z)=a_{k} z^{k}+a_{k-1} z^{k-1}+\ldots+a_{2} z^{2}+a_{1} z,
$$

convoluted with the Hilbert matrix elements in the fit order, we obtain the partial sums

$$
\bar{f}_{k}(z)=\frac{a_{k}}{k} z^{k}+\frac{a_{k}}{k-1} z^{k-1}+\ldots+\frac{a_{2}}{2} z^{2}+a_{1} z, k \geq 2, a_{1}=1 .
$$


For the above partial sums $\bar{f}_{k}(z)$, we let

$$
\begin{array}{r}
\bar{f}_{k}(z)=\frac{a_{k}}{k} z^{k}+\frac{a_{k}}{k-1} z^{k-1}+\ldots+\frac{a_{2}}{2} z^{2}+a_{1} z, \\
a_{1}=1, z \in U \\
:=b_{0} z^{k}+b_{1} z^{k-1}+\ldots+b_{k-2} z^{2}+b_{k-1} z+b_{k}, \\
b_{k-1}=a_{1}=1, b_{k}=0 .
\end{array}
$$

The minors of Hurwitz $(k \times k)$ matrix for (1), are defined by

$$
\begin{aligned}
& \Delta_{1}\left(\bar{f}_{k}\right)=\left|b_{1}\right| \\
& \Delta_{2}\left(\bar{f}_{k}\right)=\left|\begin{array}{ll}
b_{1} & b_{3} \\
b_{0} & b_{2}
\end{array}\right| \\
& \Delta_{3}\left(\bar{f}_{k}\right)=\left|\begin{array}{lll}
b_{1} & b_{3} & b_{5} \\
b_{0} & b_{2} & b_{4} \\
0 & b_{1} & b_{3}
\end{array}\right| \\
& \vdots
\end{aligned}
$$

Definition 2.1 For $z \in U$, the polynomial $\bar{f}_{k}(z)$ is called stable, asymptotically stable and unstable if and only if $\Delta_{j}>0, \Delta_{j}=0, \Delta_{j}<0$, for all $j=1,2,3, \ldots$, respectively.

From (1), we define the partial sums

$$
g_{k}(z):=z+\frac{a_{k}}{k} z^{k} .
$$

We proceed to construct new classes based on $g_{k}(z)$. A computation implies

$$
\begin{aligned}
& P_{k}(z)=\frac{z g_{k}^{\prime}(z)}{g_{k}(z)}, k \geq 2 \\
& =1+\sum_{m=1}^{\infty} \frac{(-1)^{m-1}(k-1)}{k^{m}} a_{k}^{m} z^{m(k-1)} \\
& :=1+\sum_{m=1}^{\infty} \frac{(-1)^{m-1}(k-1)}{k^{m}} a_{k}^{m} w^{m}, w=z^{k-1} .
\end{aligned}
$$

Thus for $k=2,3,4, \ldots$, we have the following classes:

$$
\begin{aligned}
& P_{2}(w)=1+\sum_{m=1}^{\infty} \frac{(-1)^{m-1}}{2^{m}} a_{2}^{m} w^{m} \\
& P_{3}(w)=1+\sum_{m=1}^{\infty} \frac{2(-1)^{m-1}}{3^{m}} a_{3}^{m} w^{m},
\end{aligned}
$$

We call the above classes the coefficient $\left(a_{k}\right)$-starlike and they denoted by $S^{*}\left(a_{k}\right)$. Similarly, we define the coefficient $\left(a_{k}\right)$-convex, which denoted by $\mathcal{C}\left(a_{k}\right)$, as follows:

$$
\begin{aligned}
& Q_{k}(z)=1+\frac{z g_{k}^{\prime \prime}(z)}{g_{k}^{\prime}(z)}=1+\sum_{m=1}^{\infty}(-1)^{m-1} k a_{k}^{m} z^{m k} \\
& :=1+\sum_{m=1}^{\infty}(-1)^{m-1} k a_{k}^{m} w^{m}, \quad w=z^{k} .
\end{aligned}
$$

Thus for $k=2,3, \ldots$, we have the following classes:

$$
\begin{aligned}
& Q_{2}(w)=1+\sum_{m=1}^{\infty} 2(-1)^{m-1} a_{2}^{m} w^{m} \\
& Q_{3}(w)=1+\sum_{m=1}^{\infty} 3(-1)^{m-1} a_{3}^{m} w^{m}, \\
& \vdots
\end{aligned}
$$

In the same manner of the above classes, one can construct $a_{k}$-class such as close to convex, uniformly classes and concave. Based on these classes, we can study the stability of starlikeness as well as convexity. Moreover, relations concerning these classes can be formulated such as $H_{1}(1), H_{2}(1), \ldots$.

\section{Outcomes}

We have the following stability results for the classes $S^{*}\left(a_{k}\right)$ and $\mathcal{C}\left(a_{k}\right)$ :

Theorem 3.1 Consider $P_{2} \in S^{*}\left(a_{2}\right), \quad a_{2}>0$. Then a polynomial of degree 2 is starlike stable, while of degree 3 is not stable.

Proof. By employing $P_{2}$, in Eq. (3), polynomials of degree 2 and 3 can be expressed respectively as follows:

$$
\begin{aligned}
p_{2,2}(w) & =1+\frac{a_{2}}{2} w-\frac{a_{2}^{2}}{2^{2}} w^{2} \\
& :=b_{2}+b_{1} w+b_{0} w^{2}
\end{aligned}
$$

and

$$
\begin{aligned}
p_{2,3}(w) & =1+\frac{a_{2}}{2} w-\frac{a_{2}^{2}}{2^{2}} w^{2}+\frac{a_{2}^{3}}{2^{3}} w^{3} \\
& :=b_{3}+b_{2} w+b_{1} w^{2}+b_{0} w^{3} .
\end{aligned}
$$

Let $a_{2}>0$, thus we obtain

$$
\Delta_{1}\left(p_{2,2}\right)=\Delta_{2}\left(p_{2}\right)=\frac{a_{2}}{2}>0
$$

and

$$
\Delta_{2}\left(p_{2,3}\right)=-\frac{a_{2}^{3}}{2^{2}}<0 .
$$

Theorem 3.2 Consider $Q_{2} \in \mathcal{C}\left(a_{2}\right), \quad a_{2}>0$. Then a polynomial of degree 2 is convex stable, while of degree 3 is not stable.

Proof. Consider $Q_{2} \in \mathcal{C}\left(a_{2}\right), a_{2}>0$. Then polynomials of degree 2 and 3 can be formulated respectively as follows:

$$
\begin{aligned}
q_{2,2}(w) & =1+\frac{a_{2}}{2} w-\frac{a_{2}^{2}}{2^{2}} w^{2} \\
& :=b_{2}+b_{1} w+b_{0} w^{2}
\end{aligned}
$$

and 


$$
\begin{aligned}
q_{2,3}(w) & =1+\frac{a_{2}}{2} w-\frac{a_{2}^{2}}{2^{2}} w^{2}+\frac{a_{2}^{3}}{2^{3}} w^{3} \\
& :=b_{3}+b_{2} w+b_{1} w^{2}+b_{0} w^{3} .
\end{aligned}
$$

Let $a_{2}>0$, thus we obtain

$$
\Delta_{1}\left(q_{2,2}\right)=\Delta_{2}\left(q_{2}\right)=2 a_{2}>0
$$

and

$$
\Delta_{2}\left(q_{2,3}\right)=-6 a_{2}^{3}<0 .
$$

Consider $p_{n} \in S^{*}\left(a_{k}\right)$. We deal with polynomial sequences $\left(p_{n}\right) \in S^{*}\left(a_{k}\right), k \geq 2$ (partial sums) satisfying the recurrent relation

$$
\begin{gathered}
w p_{n}(w)=\rho_{n}\left(p_{n+1}(w)-p_{n-1}(w)\right), n \geq 1 \\
\left(\rho_{n}>0, n \geq 1, p_{0}=1\right)
\end{gathered}
$$

and

$$
p_{n}(w)=1+\sum_{m=1}^{n} \frac{(-1)^{m-1}(k-1)}{k^{m}} a_{k}^{m} w^{m} .
$$

Define the Turan determinant as follows:

$$
\Lambda_{n}(w)=p_{n}^{2}(w)-p_{n-1}(w) p_{n+1}(w), n \geq 1 .
$$

We shall prove inequality of the form

$$
c \leq\left|\Lambda_{n}(w)\right| \leq C, 0<|w|<1,0<c<C .
$$

Theorem 3.3 Assume $p_{n}(w)$ satisfies (6). Then

$$
\rho_{n} \Lambda_{n}+\rho_{n-1} \Lambda_{n-1}=\left(\rho_{n}-\rho_{n-1}\right)\left(p_{n}^{2}-p_{n-1}^{2}\right), n \geq 1 .
$$

Proof. By (6), we have

$$
p_{n+1}(w)=\frac{w \rho_{n}+\rho_{n} p_{n-1}}{\rho_{n}}
$$

this yields that

$$
\Lambda_{n}=p_{n}^{2}-\left(\frac{w \rho_{n}+\rho_{n} p_{n-1}}{\rho_{n}}\right) p_{n-1} .
$$

Consequently, we obtain

$$
\rho_{n} \Lambda_{n}=\left(\rho_{n}-\rho_{n-1}\right) p_{n}^{2}+\rho_{n-1} \rho_{n} \rho_{n-2}-\rho_{n} \rho_{n-1}^{2}
$$

By the definition of $\Lambda_{n}$, we conclude that

$$
\begin{aligned}
& \rho_{n-1} \Lambda_{n-1}(w) \\
& =\rho_{n-1} \rho_{n-1}^{2}(w)-\rho_{n-1} \rho_{n-2}(w) p_{n}(w), n \geq 1 .
\end{aligned}
$$

then summing (9) and (10), we arrive at the desired assertion. This completes the proof.

Theorem 3.4 Let $\left(\rho_{n}\right) n \geq 1$ be increasing sequence. If $\frac{\rho_{n}-\rho_{n-1}}{\rho_{n}}>1$ then

$$
\left|\Lambda_{n}(w)\right|>0, w \in U \backslash\{0\}, n \geq 1
$$

Proof. It suffices to show that $\left|\Lambda_{1}(w)\right|>0$. By the proof of Theorem 3.3 and the fact that $p_{0}=1$ and $p_{-1}=0$, we conclude that

$$
\begin{aligned}
\left|\Lambda_{1}(w)\right| & =\left|\frac{\left(\rho_{1}-\rho_{0}\right)}{\rho_{1}} p_{1}^{2}-p_{0}^{2}\right| \\
& =\left|\frac{\left(\rho_{1}-\rho_{0}\right)}{\rho_{1}} p_{1}^{2}-1\right| \\
& >\left|p_{1}^{2}-1\right| \geq 0 .
\end{aligned}
$$

Therefore, by the assumptions of the theorem, we have $\left|\Lambda_{1}(w)\right|>0$. Hence by induction we obtain $\left|\Lambda_{1}(w)\right|>0$, $n \geq 1$.

Define a function $g_{n}(w):=p_{n+2}-p_{n}$ then $g_{n}(w)$ satisfies the following property :

Proposition 3.1 For $n \geq 1$ we have

$$
w g_{n}(w)=\rho_{n+2} g_{n+1}(w)-\rho_{n} g_{n-1}(w) .
$$

Proof. A calculation implies that

$$
\begin{aligned}
& \rho_{n+2} g_{n+1}(w)-\rho_{n} g_{n-1}(w) \\
& =\rho_{n+2} p_{n+3}(w)-\rho_{n+2} p_{n+1}(w) \\
& =w p_{n+2}(w)-\rho_{n} p_{n+1}(w)+\rho_{n} p_{n-1}(w) \\
& =w g_{n}(w)+w p_{n}-\rho_{n} p_{n+1}(w)+\rho_{n} p_{n-1}(w) \\
& =w g_{n}(w) .
\end{aligned}
$$

Theorem 3.5 For $n \geq 1$ we have

$$
w^{2} \Lambda_{n}(w)=\rho_{n}^{2} g_{n-1}^{2}(w)-\rho_{n+1} \rho_{n-1}\left(g_{n-2}(w) g_{n}(w)\right),
$$

where $g_{n}(w)=p_{n+2}(w)-p_{n}(w), \quad n \geq 1$.

Proof. We observe that

$$
\rho_{n} g_{n-1}(w)=\rho_{n}\left(p_{n+1}(w)-p_{n-1}(w)\right)=w p_{n},
$$

and

$$
\begin{aligned}
& \rho_{n+1} \rho_{n-1} g_{n-2}(w) g_{n}(w) \\
& =\rho_{n-1}\left(p_{n}(w)-p_{n-2}(w)\right) \rho_{n+1}\left(p_{n+2}(w)-p_{n-1}(w)\right)(12) \\
& =w^{2} p_{n-1} p_{n+1} .
\end{aligned}
$$

Subtracting (12) from (11), we conclude the desired assertion.

Theorem 3.6 Consider that $\left(p_{n}\right)$ achieves (6) with $\rho_{0}<1$. Let $\left(\rho_{n}\right), n \geq 1$ be increasing such that $\rho_{n} \leq 1 / 2$ and

$$
\rho_{n}-\rho_{n-1} \geq \frac{\rho_{n}}{1-\rho_{n}}\left(\rho_{n+1}-\rho_{n}\right), n \geq 1
$$

Then

$$
\left|\Lambda_{n}(w)\right| \geq c, c>0, w \in U, n \geq 1 .
$$

Proof. Clearly that (13) is equivalent to $\left(\rho_{n}^{2}\right)$ being increasing. Define the formula 


$$
A_{n}(w):=\rho_{n} g_{n-1}^{2}(w)-\rho_{n+1} g_{n-2}(w) g_{n}(w) .
$$

Since $\rho_{n-1} \leq \rho_{n}$, therefore, in view of Theorem 3.5, we obtain

$$
\left|\Lambda_{n}(w)\right| \geq \rho_{n-1}\left|\Lambda_{n}(w)\right|, w \rightarrow \pm 1 .
$$

By Proposition 3.1, we have the following expression :

$$
\begin{aligned}
A_{n}(w)= & \rho_{n-1} g_{n-2}^{2}(w)+\rho_{n} g_{n-1}^{2}(w) \\
& +w g_{n-2}(w) g_{n-1}(w) .
\end{aligned}
$$

Multiplying Eq.(15) by $\frac{\rho_{n-1}}{\rho_{n+1}}$ and replacing $n$ by $n-1$, we arrive on

$$
A_{n}(w)-\frac{\rho_{n-2}}{\rho_{n}} A_{n-1}(w)=\frac{\rho_{n-1} \rho_{n}-\rho_{n-2} \rho_{n-1}}{\rho_{n}} g_{n-2}^{2}(w) .
$$

Consequently, we conclude that

$$
\left|A_{n}-\frac{\rho_{n-2}}{\rho_{n}} A_{n-1}\right| \geq 0 .
$$

By iterating the quantities $A_{n}$ and $A_{n-1}$, we attain in

$$
\left|A_{n}(w)\right| \geq \frac{\rho_{1} \ldots \rho_{n-2}}{\rho_{3} \ldots \rho_{n}}\left|A_{2}\right| .
$$

But by utilizing Eq.(15) and Eq.(16), we find

$$
\left|A_{2}(w)\right|=\left|\rho_{1} g_{0}^{2}+\rho_{2} g_{1}^{2}-g_{0} g_{1}\right| \geq \frac{\rho_{1}}{\rho_{1}^{2}}=\frac{1}{\rho_{1}} .
$$

Therefore, (14) becomes

$$
\left|A_{n}(w)\right| \geq \frac{\rho_{1} \ldots \rho_{n}}{\rho_{1} \ldots \rho_{n}} \frac{\rho_{1} \rho_{2}}{\rho_{n} \rho_{1}}=\frac{\rho_{2}}{\rho_{n}}:=c .
$$

Hence the proof.

Theorem 3.7 Consider that $\left(p_{n}\right)$ achieves (6) with $p_{0}<1$. Let $\left(p_{n}\right), n \geq 1$ be decreasing such that $\rho_{n} \geq 1 / 2$ and

$$
\rho_{n}-\rho_{n-1} \leq \frac{\rho_{n}}{1-\rho_{n}}\left(\rho_{n+1}-\rho_{n}\right), n \geq 2
$$

Then

$$
\left|\Lambda_{n}(w)\right| \leq C, C>0, w \in U, n \geq 2 .
$$

Proof. By letting $\Upsilon_{n}:=\sqrt{\rho_{n+1} \rho_{n}}$, with the following properties:

- $\lim \Upsilon_{n}=\frac{1}{2}$

- $\Upsilon_{n+1}-\Upsilon_{n}=\frac{\rho_{n+2}\left(\rho_{n+1}-\rho_{n}\right)+\rho_{n}\left(\rho_{n+2}-\rho_{n+1}\right)}{\sqrt{\rho_{n+2}+2 \rho_{n+1}}+\sqrt{\rho_{n+1} \rho_{n}}}$

- $\sum_{n=1}^{\infty}\left|\Upsilon_{n+1}-\Upsilon_{n}\right|<\infty$.

The last property is valid by the monotonicity of $\left(\rho_{n}\right)$ in (17). Define a polynomial $\left(P_{n}\right)$ by utilizing $\Upsilon_{n}$ as follows: for

$$
P_{n}(w)=\varrho_{n} p_{n}(w), w \in \bar{U}
$$

where

$$
\varrho_{n}:=\sqrt{\frac{\rho_{0}}{\rho_{n}}}, \quad \varrho_{0}=1, \quad n \geq 1
$$

satisfying

$$
P_{n}=\Upsilon_{n} P_{n+1}+\Upsilon_{n-1} P_{n-1} .
$$

Obviously, $P_{n}$ satisfies

$$
\lim _{n \rightarrow \infty}\left[P_{n}^{2}-P_{n-1}(w) P_{n+1}(w)\right]<\infty .
$$

This implies that $\left(P_{n}(w)\right), \quad w \in \bar{U}$ is uniformly bounded on a compact set for $n \rightarrow \infty$. By the definition of the Turan determinant, we obtain

$$
\Lambda_{n}(w)=\frac{1}{\varrho_{n}^{2}}\left(P_{n}^{2}(w)-\lambda_{n} P_{n-1}(w) P_{n+1}(w)\right),
$$

where

$$
\lambda_{n}=\frac{\varrho_{n}^{2}}{\varrho_{n-1} \varrho_{n+1}},
$$

such that

$$
\lim _{n \rightarrow \infty} \lambda_{n}=1
$$

We conclude that there exists a constant $C \in(0, \infty)$ such that

$$
\left|\Lambda_{n}(w)\right| \leq C, n \geq 1, w \in \bar{U} .
$$

Remark 3.1 If $\rho_{0}=1$ in Theorems 3.6 and 3.7, we obtain that the coefficient $a_{k}=0$. For example, if $\left|a_{k}\right|<k$ (starlike class), then $\rho_{0} \leq 1 / k, k \geq 2$. Thus, Theorem 3.7 implies that $\left|\Lambda_{n}\right| \leq \rho_{0}<1$ as $w \rightarrow+1$. Moreover, the above results can be considered for a sequence of polynomials $\left(q_{n}\right) \in \mathcal{C}\left(a_{k}\right), k \geq 2$.

\section{Applications}

In this section, we utilize the Turan determinant to fined the coefficients bound of the classes $S^{*}\left(a_{k}\right)$ and $\mathcal{C}\left(a_{k}\right)$. We have the following propositions.

Proposition 4.1 Consider the classes $S^{*}\left(a_{2}\right)$ and $S^{*}\left(a_{3}\right)$. Then $\left|a_{2}\right| \leq 1.3$ and $\left|a_{3}\right| \leq \frac{3}{2}$.

Proof. By utilizing $\Lambda_{1}$ and $\Lambda_{2}$ respectively for finding the upper bound of $\left|a_{2}\right|$ and $\left|a_{3}\right|$ A computation implies that

$$
\Lambda_{2}(w)=\frac{a_{2}^{2}}{4} w^{2}-\frac{a_{2}^{3}}{4} w^{3}
$$

and 


$$
\Lambda_{3}(w)=\frac{6}{9} a_{3}^{2} w^{2}+\frac{4}{27} a_{3}^{3} w^{3}-\frac{21}{81} a_{3}^{4} w^{4}+\frac{16}{243} a_{3}^{5} w^{5} .
$$

In view of Remark 3.1, we conclude that

$$
\begin{aligned}
\left|\Lambda_{2}(w)\right| & =\left|\frac{a_{2}^{2}}{4} w^{2}-\frac{a_{2}^{3}}{4} w^{3}\right| \\
& =\left|\frac{a_{2}^{2}}{4}-\frac{a_{2}^{3}}{4}\right|, \quad w \rightarrow \pm 1 \\
& <1 \text {, when }\left|a_{2}\right|<1.3 .
\end{aligned}
$$

Similarly for $\left|a_{3}\right|$

In the similar manner of Proposition 4.1, we have the following result:

Proposition 4.2 Consider the classes $\mathcal{C}\left(a_{2}\right)$. Then $\left|a_{2}\right| \leq \frac{1}{2}$

Proof. By utilizing $\Lambda_{2}:=q^{2}(w)-q_{1}(w) q_{3}(w)$, we obtain

$$
\left|\Lambda_{2}(w)\right|=\left|2 a_{2}^{2} w^{2}-2 a_{2}^{3} w^{3}\right|
$$

which implies that $\left|\Lambda_{2}(w)\right|<1$ when $\left|a_{2}\right| \leq \frac{1}{2}$.

\section{Conclusion}

We imposed a new technique for finding the coefficients bound. This method based on several types of matrices. The major type was the Tura in the open unit disk. We proved the boundedness of this matrix from below as well as from above. We defined classes of analytic functions, depending on one coefficients, calculating by some special type of partial sums. The stability of these classes is considered by utilizing the Hurwitz matrix. We illustrated some applications of this method for two well defined classes (starlike and convex). The above method can be employed on other classes such as uniform, concave etc.

\section{Conflict of Interests}

The author declares that there is no conflict of interests regarding the publication of this article.

\section{References}

[1] P. Dienes, The Taylor Series. Dover, New York (1957).

[2] A. Edrei, Sur les dterminants rcurrents et les singularits dune fonction done por son dveloppement de Taylor. Compos. Math. 7, 20-88 (1940).

[3] D. G. Cantor, Power series with integral coefficients. Bull. Am. Math. Soc. 69, 362-366 (1963).

[4] R. Wilson, Determinantal criteria for meromorphic functions. Proc. Lond. Math. Soc. 4, 357-374 (1954).

[5] R. Vein, P. Dale, Determinants and Their Applications in Mathematical Physics. Applied Mathematical Sciences, vol. 134. Springer, New York (1999).

[6] D. Bansal, Upper bound of second Hankel determinant for a new class of analytic functions. Appl. Math. Lett. 26(1), 103-107 (2013).

[7] K. O. Babalola, On H3(1) Hankel determinant for some classes of univalent functions. Inequal. Theory Appl. 6, 1-7 (2007).

[8] R. W. Ibrahim, Bounded nonlinear functional derived by the generalized Srivastava-Owa fractional differential operator. International Journal of Analysis, 1-7 (2013).

[9] G. Szego, Zur theorie der schlichten abbilungen. Math. Ann. 100, 188-211 (1928)

[10] S. Owa, Partial sums of certain analytic functions. Int. J. Math. Math. Sci. 25(12), 771-775 (2001).

[11] M. Darus, R. W. Ibrahim, Partial sums of analytic functions of bounded turning with applications. Comput. Appl. Math. 29(1), 81-88 (2010).

[12] R. W. Ibrahim, M. Darus, Cesáro partial sums of certain analytic functions, Journal of Inequalities and Applications, 51, 1-9 (2013). 